\title{
“LA PLACE D’AUTRUP': PERSPECTIVISMO Y NORMATIVIDAD SEGÚN LEIBNIZ
}

\author{
Evelyn Vargas \\ UNLP-CONICET
}

\begin{abstract}
RESUMEN: Mi propósito en este artículo es analizar una forma de perspectivismo entendido como un tipo de escepticismo, y los argumentos de Leibniz en contra de él. Discuto cómo esta forma de perspectivismo conduce a la isostheneia y a la suspensión del juicio, y presento la estrategia de Leibniz para impedir estas consecuencias escépticas, y finalmente ofrezco un ejemplo de esta estrategia.
\end{abstract}

PALABRAS CLAVE: Leibniz; perspectivismo; escepticismo.

\begin{abstract}
My purpose in this paper is to analyse a form of perspectivism understood as a kind of skepticism and Leibniz's arguments against it. I discuss how, according to him, this form of perspectivism leads to isostheneia and suspension of judgment, and I introduce Leibniz's strategy to prevent these skeptical consequences; finally I present an example of this strategy.
\end{abstract}

KEYWORDS: Leibniz; perspectivism; skepticism.

\section{Introducción}

La renovación del tema del perspectivismo en nuestra cultura ha tomado diversas formas que van desde la reflexión filosófica al reconocimiento banal de un lugar común. Aquellos pensadores que se han ocupado de la cuestión encuentran en Nietzsche su antecedente e inspiración. De entre todos ellos quisiera mencionar a Richard Rorty, quien creyó poder mostrar una cultura intelectual post epistemológica a partir de un diagnóstico crítico de la filosofía 
moderna, y su intento de autolegitimación, ${ }^{1}$ intento que encuentra su lugar en la duda escéptica característica de la epistemología cartesiana. Semejante proyecto filosófico, sostiene Rorty, depende de la viabilidad de la mente de producir pensamientos u otras representaciones que reflejen correctamente la realidad.

No es sorprendente entonces que su apreciación del perspectivismo de

Nietzsche sea también crítico:

In theory, Nietzsche is not playing this Kantian game. In practice, just insofar as he claims to see deeper rather than differently, claims to be free rather than merely reactive, he betrays his own perspectivism and his own nominalism. He thinks that his historicism will save him from this betrayal, but it does not. ${ }^{2}$

Nietzsche traiciona su propio perspectivismo y recae en la metafísica, al volverse un teórico de la voluntad de poder. ${ }^{3}$ El perspectivista es consciente de estar redescribiendo a los filósofos del pasado según alguna idea general con

1 'This is equivalent to saying that if we do not have the distinction between what is "given" and what is "added by the mind," or that between the "contingent" (because influenced by what is given) and the necessary (because entirely "within" the mind and under its control), then we will not know what would count as a "rational reconstruction" of our knowledge. We will not know what epistemology's goal or method could be' (RORTY, 1979. pp.168-169).

${ }^{2}$ Rorthy (1989, p.105).

${ }^{3}$ Escribe Rorty (1989, p. 107): "If he had been faithful to his own perspectivalism and antiessentialism, he would have avoided the temptation into which Hegel fell. That was the temptation of thinking that once you have found a way to subsume your predecessors under a general idea you have thereby done something more than found a redescription of them - a redescription which has proved useful for your own purposes of self-creation. If you go on to conclude that you have found a way to make yourself quite different from those predecessors, to do something quite different from what they did, then you are doing what Heidegger called "relapsing into metaphysics." For now you are claiming that none of the descriptions that applied to them applies to you - that you are separated from them by an abyss. You are acting as if a redescription of one's predecessors got one in touch with a power other than oneself - something capitalized: Being, Truth, History, Absolute Knowledge, or the Will to Power. This was Heidegger's point when he called Nietzsche "merely an inverted Platonist": The same urge to affiliate with somebody bigger which had led Plato to reify "Being". 
vistas a un propósito particular. De otro modo se recae en la metafísica, en la ambición de descubrir la realidad detrás de las apariencias. ${ }^{4}$

Un vocabulario, añade, es una herramienta; por tanto, un vocabulario descriptivo es útil en la medida en que los patrones que resalta son útiles para criaturas con nuestros intereses y necesidades, de modo que no hay un vocabulario privilegiado cuyo propósito sea servir como piedra de toque de nuestras diversas prácticas descriptivas. La epistemología, en su rol de supervisor de dichas prácticas, no es necesaria para la cultura. Cuando el propósito es la secularización y la democratización, dicho compromiso puede promoverse mediante redescripciones y no mediante la argumentación. Rorty no solo contrapone metafísica y perspectivismo, también es crítico del rol de la argumentación para nuestro progreso intelectual. Estas ideas son centrales para su conversacionalismo.

Pero nuestras prácticas dialógicas son también centrales para Leibniz, sin que ello involucre extraer las consecuencias escépticas y antiepistemológicas que Rorty señala. Así, en lugar de intentar definir la noción de perspectiva, me

\footnotetext{
${ }^{4}$ The metaphysicians attempt to rise above the plurality of appearances in the hope that, seen from the heights, an unexpected unity will become evident - a unity which is a sign that something real has been glimpsed, something which stands behind the appearances and produces them. By contrast, the ironist canon I want to discuss is a series of attempts to look back on the attempts of the metaphysicians to rise to these heights, and to see the unity which underlies the plurality of these attempts. The ironist theorist distrusts the metaphysician's metaphor of a vertical view downward. He substitutes the historicist metaphor of looking back on the past along a horizontal axis. But what he looks back on is not things in general but a very special sort of person, writing a very special kind of book. The topic of ironist theory is metaphysical theory. For the ironist theorist, the story of belief in, and love of, an ahistorical wisdom is the story of successive attempts to find a final vocabulary which is not just the final vocabulary of the individual philosopher but a vocabulary final in every sense - a vocabulary which is no mere idiosyncratic historical product but the last word, the one to which inquiry and history have converged, the one which renders further inquiry and history superfluous. (RORTY 1989, p.96).
} 
ocuparé de describir las estrategias puestas en juego por parte de Leibniz con relación a aquellos que extraían consecuencias escépticas a partir del reconocimiento del carácter perspectivizado de la cognición humana.

\section{Perspectivismo y escepticismo}

Como es bien conocido, la modernidad temprana asistió a una renovación y transformación de la tradición escéptica clásica. Según una interpretación ampliamente aceptada de dicha transformación, el uso de los argumentos clásicos por parte de Descartes no se habría limitado a una mera extensión de su alcance sino que introduce genuina novedad: por primera vez, la cuestión de la existencia del mundo externo es puesta en tela de juicio. Condición de este problema es la consideración del propio cuerpo como parte de la realidad externa, es decir, extramental. Pero por esta misma razón, este escepticismo no puede extenderse a la vida cotidiana; el escepticismo filosófico puede aislarse de nuestras preocupaciones vitales. ${ }^{5}$

Ésta es, sin duda, una imagen muy difundida del escepticismo moderno. Una característica notable de este enfoque post-cartesiano de los argumentos escépticos clásicos es su desvinculación con los asuntos de la vida práctica; su alcance, así como su refutación, se limitan al ámbito de la discusión

\footnotetext{
${ }^{5}$ Dice al respecto Burnyeat (1982, p.39): "To sum up, it is not accident that in Descartes' philosophy the following elements are found in the closest association: hyperbolical doubt and the problem of the existence of the external world-and the refutation of the ancient skeptical tradition. All these are substantially new with Descartes, and derive from the very seriousness (in one sense) with which he took the traditional skeptical materials. It is essential here that this seriousness is entirely methodological". Un análisis pormenorizado del argumento cartesiano, y su novedad respecto de la tradición antigua en Williamson (2010).
} 
filosófica. Quisiera sugerir, en cambio, un modo de abordaje complementario al del discurso filosófico, que se vincula con el perspectivismo moderno, y que también transforma los argumentos escépticos clásicos. Para artistas y hombres de letras familiarizados con el uso de la perspectiva, las técnicas de tromp-l'oeil, anamorfismo, etc., se extienden a consideraciones acerca del valor de la cognición humana. La contemplación de un cuadro como 'El embajador' de Holbein, con su exagerado realismo ofrece una experiencia de ilusión de la que ya no resulta posible el regreso a la visión 'normal,' la perspectiva frontal que representa a dos embajadores franceses rodeados de objetos característicos del humanismo, con su evocación del poder intelectual y político ya no puede tomarse en serio. ${ }^{6}$ Esta técnica pictórica encuentra su equivalente retórico en la literatura; el autor propone múltiples puntos de vista, permitiendo lecturas que oscilan entre lo literal y lo aparentemente distorsionado, pero en tanto no ofrece una visión unificadora, ninguna de estas perspectivas determina la 'verdad' del personaje o la situación. ${ }^{7}$

En sus Bosquejos de Pirronismo Sexto Empírico describía el modo de vida escéptico como aquel que secunda las apariencias, pero suspende el juicio acerca de qué apariencias son correctas. El aprendizaje y ejercicio de un arte no involucra para un escéptico el abandono de la actitud suspensiva. ${ }^{8}$ Pero el dominio de las técnicas para producir apariencias que socaban nuestra confianza

\footnotetext{
${ }^{6}$ Incluido entre los objetos se encuentra un ejemplar del Elogio de la locura de Erasmo, donde se insiste en lo vano de todo conocimiento.

${ }^{7}$ Existen numerosos estudios al respecto; me limito a indicar uno reciente, que contiene una amplia bibliografía, Massey (2007), para el caso de la pintura, y Castillo (2001), como ejemplo de su uso literario. ${ }^{8}$ Es uno de los modos de vivir según la naturaleza (cfr. SEXTO EMPÍRICO, 1996, p. I 7).
} 
en el punto de vista 'normal' pone en evidencia el carácter artificial de la representación realista. Este perspectivismo no se limita a constatar la existencia de múltiples puntos de vista para favorecer la actitud escéptica sino que descansa en una práctica que pone de manifiesto dicha artificialidad.

\section{El lugar del otro}

El uso del modelo perspectivista por parte de Leibniz es bien conocido. Bien podría decirse que las cosas son puntos de vista pues toda sustancia se define por el punto de vista que tiene sobre el universo. La cuestión no radica para Leibniz en el reconocimiento de la multiplicidad infinita de las que las miradas humanas solo constituyen una parte, sino en su conciliación. Nuestras prácticas discursivas dialógicas no solo se limitan a poner en evidencia nuestras divergencias respecto a los asuntos sobre los que se conversa. El arte de disputar tiene por condición la diversidad de las opiniones. Pero Leibniz no fue un partidario de la insularización de la argumentación filosófica, disputar no puede ser un divertimento sin consecuencias sobre nuestra vida. Esta es la idea que el padre Eremita quiere trasmitir al Marqués de Pianese en la conversación que mantienen en este diálogo leibniciano de aproximadamente 1680. Este último representa un nuevo tipo de intelectual, que, desencantado, abandona la empresa filosófica. ${ }^{9}$

El marqués plantea la cuestión escéptica en estos términos:

${ }^{9}$ Ezequiel de Olaso (1984, pp.220-221) identifica este tipo de intelectual con el libertino, y señala que es el que preocupa a Leibniz, por las consecuencias políticas que su actitud involucra. 
Car je voy que les hommes ne demeurent presque jamais d'accord, qu'il n'y a pas moyen de sortir des doutes, et que les méditations mêmes ne servent qu'à nous embarrasser d'avantage. Il me semble que la nature ne nous a pas fait pour jouir de la vérité, mais pour nous régler sur les apparences. C'est pourquoy il y a long temps que j'ay pris la resolution, de ne me plus piquer de ces connoissances prétendues; et je me contenteray de suivre un train de vie aisée et libre de toutes les reflexions qui entestent. ${ }^{10}$

Como en Sexto, la guía de la naturaleza consiste en regirse por las apariencias, ${ }^{11}$ dejar de lado las pretensiones de conocimiento y finalmente, dejar de lado las reflexiones que producen intranquilidad al alma, en la medida que no pueden erradicar la duda. En su larga respuesta, el padre eremita comienza por reconocer los distintos puntos de vista sobre cualquier asunto, que nacen de lo que perturba a cada ser humano:

Voicy donc la cause de l'incertitude et des disputes sans fruit: pour le remede nous en parlerons par après. Il y a des commodités et des incommodités, des biens et des maux dans toutes les choses du monde sacrées et profanes, c'est ce qui trouble les hommes, c'est ce qui fait naistre cette diversité d'opinions, chacun envisageant les objets d'un certain costé: il n'y en a que tres peu qui ayent la patience de faire le tour de la chose jusqu'à se mettre du costé de leur adversaire; c'est à dire qui veuillent avec une application égale, et avec un esprit de juge desintéressé examiner et le pour, et le contre afin de voir de quel costé doit pencher la balance. ${ }^{12}$ (el subrayado es propio)

Pero el diagnóstico permite también vislumbrar la solución; en la medida en que cada parte considera el asunto en debate desde un cierto costado, se trata de 'darle la vuelta' al problema y adoptar otro punto de vista, el del adversario, sopesando sus ventajas y desventajas. Leibniz identifica además otra

${ }^{10} \mathrm{AA}$ VI, 4, p. 2249.

11 Sexto Empírico 1996, I VII, pp.22-24.

12 AA VI, 4, p.2251. 
causa de los desacuerdos; puesto que se disputa por el mero placer de discutir, el razonamiento se detiene arbitrariamente, por una respuesta ingeniosa o desconcertante, antes de alcanzar la conclusión. La levedad de esta manera de disputar resulta manifiesta pues no tiene consecuencias para la vida de quienes debaten, como cuando se alcanza una paradoja. Continúa el ermita leibniciano:

Car il faudroit bien du temps pour cela, et nos passions ou distractions ne nous en donnent gueres. Ordinairement nous sommes remplis d'un certain esprit de contradiction, et nous faisons gloire de ne rien écouter, où nous ne trouvions quelque chose à redire: nous nous estudions sur tout à nous opposer en apparence à ce que les hommes ordinaires ont coustume de juger et de souhaiter: par là nous rendons tout problématique, et puisque nous nous plaisons aux disputes, pourquoy sommes nous surpris si tout est disputable pour ceux qui s'arrestent à des considerations legeres. D'autant qu'ordinairement ce n'est pas pour profiter mais pour se divertir, qu'on raisonne. Vous avés dit vous même Monsieur, que vous voulés suivre la coustume, et cependant vous dites que vous vous plaisés aux paradoxes, ce n'est donc pas pour les suivre; les sentimens singuliers nous donnent une élevation imaginaire au dessus des autres, nous serions marris de parler comme le vulgaire, quoyque nous suivions le torrent de la corruption generale. C'est que nous ne cherchons qu'à bien parler et à paroistre, et rien de plus. Quand nous avons trouvé quelque repartie adroite ou ingenieuse, qui peut rebuter ou déconcerter celuy qui nous avance quelque proposition, quoyqu'elle soit: peut-estre utile et bien fondée; nous nous contentons de cette victoire, et nous passons à d'autres matieres, sans examiner qui a eu raison dans le fonds: au moins quand nostre interest visible n'y paroist point, car nous sommes bien aises d'avoir une defaite, qui flatte nostre paresse avec quelque apparence de raison. Tout cela vient de ce que nous ne traitons la pluspart des questions que par maniere de divertissement, ou pour la parade, et point du tout pour en former une conclusion qui puisse avoir quelque influence dans la practique de nostre vie, comme les ecoliers en philosophie disputent des vertus, des vices et des passions, sans que cela les touche en aucune façon. ${ }^{13}$ (el subrayado es propio)

${ }^{13}$ AA VI 4, p.2251. 
Así, la perseverancia de las divergencias no es inevitable: por un lado, es posible y necesario adoptar otro punto de vista, el del oponente; por otra parte, poner en cuestión todo lo que los hombres ordinarios creen, es una impostura que pone la victoria por encima de todo, o un mero divertimento sin consecuencias prácticas.

Ahora bien, el escepticismo epistemológico moderno se centra en la cuestión de la distinción entre experiencias verídicas e ilusorias; prisioneros de sus propias percepciones, estados mentales a los que solo el percipiente tiene acceso, no es claro como "faire le tour de la chose" e incorporar el punto de vista del otro.

Quisiera proponer una lectura plausible de cómo se lleva a cabo esta incorporación. La expresión "el testimonio de los sentidos" que tantas veces emplea Leibniz para referirse a la percepción, no debe verse como una mera referencia metafórica. Ahora bien, el testimonio como recurso cognitivo no se contrapone a la conformidad con nuestro conocimiento, observación y experiencia como bases distintas de nuestras inferencias no demostrativas (tal como sostiene Locke en el Essay IV, xv, 4). Si el testimonio puede proveer una premisa para un argumento artificial (es decir, según el arte de razonar) es porque es un hecho el que los testimonios coincidan y en esto consiste la conformidad con lo conocido. Escribe Leibniz:

[...] de sorte que je crois que le vray Criterion en matiere des objets des sens, est la liaison des phenomenes, c'est à dire la conexión de ce qui passe en different lieux et temps, et dans les experiences de differens hommes, qui 
sont eux mêmes les uns aux autres des phenomes très importans sur cet

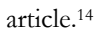

En su opúsculo "Sobre el modo de distinguir los fenómenos reales de los imaginarios", escrito probablemente entre el verano de 1683 y el invierno de 1685-6, ${ }^{15}$ Leibniz ya había sostenido que el indicio más válido de la realidad de un fenómeno es la conformidad de los propios fenómenos con lo afirmado por otras personas, aunque solo el éxito predictivo es un indicio que basta por sí mismo. ${ }^{16} \mathrm{El}$ conocimiento testimonial se genera a partir de la coherencia entre diferentes observaciones, o historias, que pueden consistir simplemente de múltiples testimonios. Si bien el testimonio ya no constituye una forma independiente de argumentación probable en los Nuevos Ensayos, Leibniz entiende que su especificidad no consiste en apoyarse en factores subjetivos como la probidad del que lo emite.

Con relación a la historia, disciplina en la que solo se cuenta con testimonios, dice Leibniz, la coincidencia entre los testimonios de distintos historiadores, en tanto sean independientes entre sí, constituyen "un gran indicio de verdad (xvi, 11)." Si los sentidos son una forma de conocimiento testimonial, sus condiciones de legitimidad epistémica son las que rigen esta práctica. Dicho

\footnotetext{
${ }^{14} \mathrm{NE}, \mathrm{AA}$ VI 6, p.374 (IV ii, 14).

15 Validissimum autem utique indicium est consensus cum tota serie vitae, maxime si idem suis quoque phaenomenis congruere alii plurimi affirment; nam alias substantias nobis similes existere non tantum probabile, sed et certum est, ut mox dicam. Sed potissimurn realitatis phaenomenorum indicium quod vel solum sufficit, est successes praedicendi phaenomena futura ex praeteritis et praesentibus, sive illa praedictio in ratione aut hypothesi hactenus succedente, sive in consuetudine hactenus observata fundetur (AA VI 4, pp.1498-1499).

${ }^{16}$ Por una parte, los indicios sólo confieren probabilidad, y afirmar lo contrario de lo que se considera probable, no implica contradicción.
} 
de otro modo, respecto de nuestros juicios perceptuales, su justificación no consiste en un contenido incorregible y privado del que el juicio constituye su formulación proposicional.

La propuesta leibniciana, por tanto, evita la adhesión incondicional tanto al fundacionalismo epistemológico como al coherentismo. Como el fundacionalista, reconoce la relevancia de nuestras experiencias para la justificación epistémica, pero la relación de apoyo evidencial no es unidireccional. Los perceptos no son contenidos cognitivos per se sino en la medida en que son puestos en relación con otros elementos cognitivos; y si no son los fundamentos en el orden de la justificación, la cuestión no se plantea en términos de la adecuación de estos ítems singulares, que operan como intermediarios entre la mente y las cosas externas. La percepción es, a la vez, directa e interpretativa pues percibimos las cosas y sucesos del mundo que nos rodea, y está entretejida en una trama de creencias. Más aun, la evidencia sensible en cuanto testimonial tiene, al igual que otras formas de justificación testimonial, las características de la conducta controlada, y por tanto, su carácter evaluativo, es decir, normativo es su condición necesaria para que la práctica tenga lugar.

En suma, Leibniz pudo rechazar el perspectivismo entendido como una forma de escepticismo. La manifestación contemporánea de dicha orientación del pensamiento encuentra en Rorty a su principal portavoz, para quien la salida de la insularización es también un abandono de la argumentación filosófica por el conversacionalismo. En la modernidad temprana, artistas y hombres de letras hicieron uso de técnicas como el anamorfismo, que mediante 
la proliferación de los puntos de vista dentro de la obra ponían en cuestión las autoridades heredadas y la legitimidad de un único punto de vista. Los temas escépticos de la equipolencia de todas las opiniones y la consiguiente suspensión del juicio son retomados y, para muchos, parecen ser la única alternativa frente a la indecidibilidad de las controversias. He considerado en primer término el diagnóstico que Leibniz lleva a cabo respecto a esta orientación, una estrategia que propone para su desactivación, y una ejemplificación de dicha estrategia. El perspectivismo en tanto reconocimiento de múltiples puntos de vista respecto de un objeto o cuestión no implica que nos encontremos en la situación de ser incapaces de decidir a favor de una posición; más aun, es adoptando un punto de vista, el del adversario, que seremos capaces de tomar partido de manera razonable, una vez consideradas sus razones. En las cuestiones no demostrativas, y en las que intervienen los sentidos, en particular, no solo es posible sino necesario considerar otros puntos de vista. Pero por sobre todo, y como lo testimonia su obra, la confrontación con otros puntos de vista es para Leibniz constitutiva del desarrollo de su propia filosofía.

\section{Ediciones de escritos de Leibniz}

LEIBNIZ, G.W. Sämtliche Schriften und Briefe, herausgegeben von der Deutschen Akademie der Wissenschaften. Darmstadt, 1923; Leipzig, 1938; Berlin, 1950 y prosigue. 


\section{BIBLIOGRAFÍA}

BURNYEART, M. F. "Idealism and Greek Philosophy: what Descartes saw and Berkeley missed." The Philosophical Review, vol. 91, 1982, pp.3-40.

CASTILlO D. (A)wry Views. Anamorphosis, Cervantes, and the Early Picaresque, West Lafayette: Purdue University Press, 2001.

DE OLASO, E. "Leibniz y el escepticismo," Revista Latinoamericana de Filosofía, vol. X, No. 3, 1984, pp.197-229.

LOCKE, J. An Essay Concerning Human Understanding. NIDDITCH, P. (ed. with Intr.). Oxford: Oxford University Press, 1975.

MASSEY, L. Picturing Space, Displacing Bodies: Anamorphosis in Early Modern Theories of Perspective. University Park: Penn State University Press, 2007.

RORTY, R. Philosophy and the Mirror of Nature, Princeton: Princeton University Press 1979. . Contingency, Irony, and Solidarity. Cambridge: Cambridge University Press, 1989.

SEXTO EMPÍRICO. Hipotiposis pirrrónicas, Madrid: Akal, 1996.

WILLIAMS, M. "Descartes' Transformation of the Sceptical Tradition". En BETT, R. (ed.), The Cambridge Companion to Ancient Scepticism. Cambridge: University Press, pp.288-313, 2010. 